\section{ORIGINAL RESEARCH}

Z. Kulcsár

C. Bonvin

V.M. Pereira

S. Altrichter

H. Yilmaz

K.O. Lövblad

R. Sztajzel

D.A. Rüfenacht

\title{
Penumbra System: A Novel Mechanical Thrombectomy Device for Large-Vessel Occlusions in Acute Stroke
}

BACKGROUND AND PURPOSE: Large IC artery occlusion is often resistant to recanalization. We present our initial experience with the PS.

MATERIALS AND METHODS: Presenting with a severe acute ischemic stroke, the first 27 consecutive patients were considered for thromboaspiration therapy and retrospective data base analysis. All patients received standard thrombectomy treatment as monotherapy or in combination with thrombolysis or IC stent placement. The primary end point was revascularization of the target vessel to grade 2 or 3 on the $\mathrm{TICl}$ scale. Secondary end points were improvement of $>4$ points on the NIHSS score at discharge and favorable outcome, and improvement in overall mortality at 3 months and in $\mathrm{s} \mathrm{CH}$ - and procedure-related adverse events.

RESULTS: At baseline, the mean age was $66 \pm 14$ years and the mean NIHSS score was $14 \pm 7$. The anterior circulation was affected in 23 patients, and there were 4 basilar artery occlusions. Intracranial stent placement was performed in 4 patients. A recanalization to $\mathrm{TICl} 2$ or 3 was achieved in 25 patients (93\%). None of the patients developed $\mathrm{SICH}$. At hospital discharge, 15 patients $(56 \%)$ had an NIHSS improvement of $>4$ and 13 patients (48\%) had an mRS score of $<2$ at 3 months. There was a significant correlation between complete vessel recanalization and favorable outcome. The all-cause mortality at 3 months was $11 \%$.

CONCLUSIONS: The PS showed a high potential for recanalization of acute thromboembolic occlusions of the large cerebral arteries. Complete recanalization was strongly correlated with good clinical outcome.

\begin{abstract}
ABBREVIATIONS: ECASS = European Cooperative Acute Stroke Study; $\mathrm{HI}=$ hemorrhagic infarctions; $I A=$ intra-arterial; $I C=$ intracranial; $I C A=$ internal carotid artery; $I Q R=$ interquartile range; $\mathrm{IV}=$ intravenous; $\mathrm{MCA}=$ middle cerebral artery; $\mathrm{MERCI}=$ Mechanical Embolus Removal in Cerebral Ischemia; $\mathrm{mRS}=$ modified Rankin Scale; NIHSS = National Institutes of Health Stroke Scale; $\mathrm{PH}=$ parenchymal hemorrhage; PROACT = Prospective Randomized On-X Valve Anticoagulation Clinical Trial; PS = Penumbra System; rtPA = recombinant tissue plasminogen activator; $\mathrm{sICH}=$ symptomatic intracerebral hemorrhage; $\mathrm{TICl}=$ Thrombolysis in Cerebral Infarction
\end{abstract}

$\mathbf{T}$ hromboembolic occlusion of major cerebral arteries is usually a very serious condition. It is commonly associated with significant neurologic deficit, poor outcome, and a high mortality rate. Such lesions are often refractory to IV and/or IA thrombolysis. ${ }^{1-4}$ Furthermore, early reocclusion after IV thrombolysis is not infrequent, which often results in clinical deterioration leading to poor outcome. ${ }^{5}$ The impact of rapid revascularization on clinical outcome is increasingly recognized..$^{6,7}$

With the advent of endovascular therapy, a number of alternative mechanical approaches have shown promise in restoring blood flow in an occluded artery causing symptoms of acute ischemic stroke. The MERCI thrombectomy device proved to be safe and effective in providing revasculariza-

\section{Received May 29, 2009; accepted after revision September 15.}

From the Neurointerventional Division (Z.K., V.M.P., H.Y., K.O.L., D.A.R.), and Neurology Division (C.B., R.S.), Department of Clinical Neurosciences; and Diagnostic Neuroradiology Division (S.A., K.O.L.), Department of Radiology, Geneva University Hospital, Geneva Switzerland; and Klinik Hirslanden (D.A.R.), Neurozentrum, Zurich, Switzerland.

Please address correspondence to Zsolt Kulcsár, MD, Klinik Hirslanden, Neurozentrum, Witellikerstr 40, 8032 Zurich, Switzerland; e-mail: kulcsarzsolt22@gmail.com

Indicates open access to non-subscribers at www.ajnr.org

DOI 10.3174/ajnr.A1924 tion. ${ }^{8,9}$ Use of IC stents for revascularization showed some promising results but also posed significant treatment-related concerns. $^{10,11}$

The PS (Penumbra, Alameda, California) is a new mechanical thrombectomy device, specifically designed to remove the thrombus in acute ischemic stroke secondary to large-vessel thromboembolism. It has 3 main components: a reperfusion catheter $(0.041,0.032$, and 0.028 inches $)$ with a separator and a thrombus-removal ring (the latter was not applied in this series of patients). In a recently published multicenter trial, the use of the PS provided excellent revascularization results. ${ }^{12}$ We present our initial experience with this system in the setting of acute thromboembolic occlusions of large cerebral arteries.

\section{Materials and Methods}

From the local stroke protocol data base covering the period between March and September 2008, a retrospective analysis was performed. We included consecutively treated patients with acute stroke who presented at our stroke center with large intracerebral artery occlusion, namely with the thromboembolism of the ICA, the M1 and M2 segments of the MCA, and the basilar artery. Patients were eligible for treatment on the basis of neurologic status and/or brain perfusion deficit as shown by CT perfusion data. The pretreatment diagnostic 
protocol consisted of a native CT scan, a CT perfusion study, and CT angiography. Patients presenting within the 3-hour time window from symptom onset were considered for systemic thrombolysis. These patients received half of the weight-adjusted full dose of IV rtPA (Actilyse, Boehringer Ingelheim, Ingelheim am Rhein, Germany) in a 30-minute period. For patients not responding to initial IV thrombolytic therapy or presenting in a time window ineligible for such treatment by policy of our local stroke center, we requested consent for IA revascularization procedures. After confirming angiographically persistent occlusion of the IC main vessel segments (carotid artery, M1 and M2 segment of the MCA, and the vertebrobasilar system), IC vascular revascularization by the PS was attempted in 27 consecutive patients. Since it became commercially available, the PS was used as a first-line IA therapeutic tool in this clinical setting.

The following information was extracted and analyzed from the local stroke protocol data base: demographics (age, sex, cardiovascular risk factors), clinical data (etiology of stroke, time of symptom onset, symptom-onset-to-treatment time, time needed from symptom onset to achieve revascularization [TICI 2 or 3], baseline and discharge NIHSS scores, 3-month mRS scores), and radiographic and angiographic data (location and extension of clot, CT perfusion data, pharmacologic treatment and mechanical devices used for revascularization, posttreatment intracerebral hemorrhage).

All the vessels showed a complete occlusion at baseline. The degree of revascularization was classified on the basis of the TICI perfusion categories, according to which the rate of perfusion is divided into 3 categories (grade 0 , no perfusion; grade 1, penetration with minimal perfusion; grade 2, partial perfusion, which is subdivided to 2a for partial filling [less than two-thirds] of the entire vascular territory and $2 \mathrm{~b}$ for complete but slower filling of all of the expected vascular territory; and grade 3 , no flow constraint and complete perfusion). ${ }^{13}$ The occurrence of intracerebral hemorrhage was verified by follow-up CT and/or MR imaging studies and was assessed according to the ECASS II criteria as being symptomatic or asymptomatic. ${ }^{14}$ Clinical and functional outcomes were assessed by the NIHSS scale score at hospital discharge and by the mRS at 3 months. ${ }^{14}$ We defined favorable clinical outcome as being mRS $0-2$, and significant neurologic improvement as being $\geq 4$-point reduction on the NIHSS scale or complete recovery (NIHSS, 0 or 1 ).

\section{Procedural Technique}

With the patient under general anesthesia, a standard $8 \mathrm{~F}$ transfemoral approach was used. To protect patients from procedural thromboembolic events as an additional element of the angiographic protocol, periprocedural heparin for systemic anticoagulation with an initial bolus dose of $2000 \mathrm{IU}$ was administered, followed by $1000 \mathrm{IU}$ injected every 60 minutes. The flush solution of the catheter system was not heparinized. The anticoagulation status of the patients was not monitored during the intervention. Initial parenchymography assessment and selective study of the concerned cerebral artery allowed rapid evaluation of the occlusion site, brain parenchymal perfusion deficit, and presence of a collateral supply. ${ }^{15}$ For IA revascularization, the PS was used as first-line therapy.

For the intervention, a triple or quadruple telescopic coaxial catheter system was built to provide mechanical support. The triple coaxial system consisted of 3 catheters. The outermost $8 \mathrm{~F}$ sheath-catheter was an 80-cm-long Vista Brite Tip (Cordis, Warren, New Jersey) positioned in the common carotid or subclavian artery; an intermediate soft-tip $6 \mathrm{~F}$ guiding catheter was positioned in the ICA or vertebral artery; and finally, the innermost device, namely the reperfusion cath- eter, targeted the IC lesion. The 0.041-inch reperfusion catheter was guided to the lesion site, most of the time over a Prowler Select Plus microcatheter (Cordis) to facilitate the advancement. If thromboaspiration failed or was considered insufficient, the same Prowler Select Plus microcatheter was used for IA rtPA injection or for deployment of an IC self-expanding Enterprise stent (Cordis).

Once the appropriate tip position of the reperfusion catheter reached the proximal end of the clot, the inner microcatheter and guidewire were removed and the separator was advanced in the reperfusion catheter. The reperfusion catheter was then connected to the aspiration pump, which generated a vacuum of -20 inches $\mathrm{Hg}$. A continuous aspiration-debulking process was facilitated by advancing and withdrawing the separator through the reperfusion catheter into the proximal end of the clot and by advancing the tip of the reperfusion catheter stepwise. If after $6-8$ passages of aspiration through the occluded area, revascularization was not achieved or was considered insufficient, local rtPA was injected in a fractionated manner. In case of an insufficient revascularization result by both of these manipulations, both procedure types were repeated at the discretion of the performing physician and by using rtPA up to half of the bodyweight-adjusted dose or a maximum of $31 \mathrm{mg}$.

Complementary stent treatment was considered and performed under 2 conditions. In complete or subtotal occlusion of the cervical origin of the ICA, carotid angioplasty and stent placement were performed at this level. Intracranial stent placement was performed in resistant IC thromboembolic occlusions or concomitant atherosclerotic stenosis at the occlusion site. In cases of stent use, additional thromboembolic protection medication was used. Body-weight-adjusted IV tirofiban infusion (Aggrastat; Medicure, Winnipeg, Manitoba, Canada) was started immediately before stent deployment and continued for 12 hours. At this time, brain CT was performed to exclude intracerebral bleeding, and the antiplatelet therapy was then continued with oral aspirin (100 mg/day) and clopidogrel $(75 \mathrm{mg} /$ day) treatment. Clopidogrel treatment was started with a loading dose of $300 \mathrm{mg}$.

Postprocedural imaging included CT or MR imaging studies performed in all patients. A nonenhanced CT was performed immediately after the endovascular intervention to exclude intracerebral hemorrhage. Follow-up CT or MR imaging or both were also performed in all patients during the hospital stay.

\section{Statistical Analysis}

Statistical evaluation was performed as appropriate by the Fisher exact test or the Pearson $\chi^{2}$ test for categoric data and the Mann-Whitney $U$ test for ordinal and interval data. A $P$ value of .05 was used to determine a statistically significant difference. All data were analyzed by SPSS software, Version 17.0 (SPSS, Chicago, Illinois).

\section{Results}

Altogether 27 consecutive patients (mean age, $66 \pm 14$ years; 13 women) presenting with acute stroke caused by large thromboembolic cerebral vessel occlusion were treated with the Penumbra thrombo-aspiration system between March and September 2008 at our stroke center. At presentation, the mean baseline NIHSS score was $14 \pm 7$. Regardless of the initial neurologic status, all patients demonstrated occlusion of a large cerebral vessel, associated with an important perfusion deficit as assessed by the CT, CT angiography, and CT perfusion studies. Among the 27 cases, 23 occlusions were in the anterior and 4 were in the posterior circulation (Table 1). 


\begin{tabular}{lc}
\hline $\begin{array}{l}\text { Table 1: Patient demographics, baseline stroke score, and site of } \\
\text { vascular occlusion }\end{array}$ \\
\hline No. of patients & 27 \\
Age (yr), mean & $66 \pm 14$ \\
Female (\%) & $13(48)$ \\
Baseline NIHSS, mean & $14 \pm 7$ \\
Site of vascular occlusion & \\
ICA/ICA terminal bifurcation (\%) & $5(19)$ \\
MCA M1 segment (\%) & $16(59)$ \\
MCA M2 segment (\%) & $2(7)$ \\
Vertebral/basilar (\%) & $4(15)$ \\
\hline
\end{tabular}

All of the target vessels were completely occluded (TICI grade, 0 ) before IA treatment with the PS was initiated. Of the 27 patients treated with the PS, 10 patients (37\%) received no thrombolytic therapy. Systemic IV rtPA therapy was performed in 13 (48\%), and IA rtPA was used in 13 patients (48\%). Combined used of systemic IV and IA rtPA was performed in 9 patients (33\%).

\section{Revascularization}

The mean time from symptom onset to the first angiographic series, namely the previously mentioned parenchymography, was 4 hours 26 minutes.

IA rtPA treatment was used with a median dose of $11 \mathrm{mg}$ (range, 5-31 mg) given as fractionated boluses alternately with the aspiration process. The main reasons for using rtPA were partial ineffectiveness of the aspiration- or treatment-associated clot fragmentation and distal migration of the clot that was deemed not accessible for further aspiration by the reperfusion system. Cervical carotid artery angioplasty was performed in 3 patients and was completed with stent placement in 2 patients. Complementary IC angioplasty with stent placement was performed in 4 patients, 2 times in the anterior circulation and 2 times in the basilar artery. In 3 of these 4 patients, IC stent placement was used for underlying atherosclerotic stenosis treatment and in 1 patient to successfully revascularize an $\mathrm{M} 2$ branch not accessible by the aspiration system.

The mean time needed to revascularization from the initial parenchymography was $97 \pm 37$ minutes, including diagnostic runs and PS preparation. The mean time from symptom onset to revascularization was 6 hours and 5 minutes.

By the end of the procedure, 5 patients demonstrated a revascularization grade of TICI $2 \mathrm{a}(19 \%) ; 6$, of $2 \mathrm{~b}(22 \%)$; and 14, of TICI 3 (52\%) (Figs 1 and 2); no recanalization could be achieved in 2 patients (7\%) (Table 2 ). There was no significant difference in the final revascularization rate between the groups with and without IA rtPA use. Comparing the subgroups of patients with and without prior IV thrombolysis (13 and 14 patients, respectively), we found more patients achieving complete recanalization in the group without IV thrombolysis ( 5 and 9 patients, respectively). The 2 patients in whom no revascularization could be achieved belonged to the group with previous IV rtPA therapy.

There were 6 patients in the study group who received thrombectomy treatment with the PS alone, without any additional IV or IA thrombolytic therapy or cervical carotid or IC stent placement. This subgroup was more disabled compared with the whole study group, presenting with a baseline
NIHSS score of 17 . In this subgroup, 4 patients had a recanalization grade of TICI 3; 1 , of TICI $2 \mathrm{~b}$; and 1 , of TICI $2 \mathrm{a}$. The mean procedure time and the mean onset to recanalization time in this subgroup were similar to those in the whole study group (95 versus 97 minutes and 365 versus 348 minutes, respectively). Despite the satisfying recanalization results, the outcome was less favorable, with only 2 patients showing a favorable resolution. Two of the patients from the whole series who died belonged to this subgroup. They were 91 and 83 years of age, with initial NIHSS scores of 17 and 24, respectively.

Clot fragmentation due to the mechanical manipulations or thrombolysis effects and distal migration were observed in 3 patients. Two of these patients had initially an M1 occlusion; and one of them, a proximal M2 branch occlusion. The combination of thrombectomy and IA thrombolysis resulted in complete local revascularization in each. The control angiogram performed after the reopening of the main occlusion site, however, demonstrated the embolic occlusion of a distal M3 branch, which was considered not amenable to further revascularization attempts in both patients.

Procedural adverse events were registered in 3 patients $(11 \%)$. One patient had subarachnoid hemorrhage visualized by contrast material extravasation. This was caused most probably by a vessel perforation with the separator tip of the reperfusion system at the level of occlusion. The hemorrhage did not result in any deterioration of the subject's neurologic condition and resolved with no additional action taken. In the second patient with a proximal M1 segment occlusion, the M1 segment was completely recanalized with thromboaspiration and IA thrombolysis; however, the occlusion of the proximal M2 segment persisted despite manipulations. The separator was difficult to advance through the occlusion site and to retrieve in the reperfusion catheter, suggesting a relatively hard consistency occlusive clot. By applying an excessive force during retrieval, the soft wire tip of the separator broke and was lost in the thrombus of the M2 branch, which remained occluded. Despite this event, in this patient, the final revascularization was of TICI $2 \mathrm{a}$ degree. In the third patient, during catheterization, we encountered a reperfusion catheter disintegration at its junction to a more proximal portion. The distal part of the separated catheter could be removed without further complications. We believe that these procedural adverse events did not have any significant deleterious clinical effect on these patients' outcomes.

\section{Clinical Outcome}

The mean NIHSS score at hospital discharge was $7 \pm 6$, and $56 \%$ of patients $(n=15)$ showed an improvement of $\geq 4$ points on this scale. At 90 days postprocedure, 13 patients $(48 \%)$ had a good functional outcome $(\mathrm{mRS}, \leq 2)$ and 7 patients $(26 \%)$ had a bad outcome (mRS, 5 or 6$)$. According to the ECASS II study definition criteria for symptomatic and asymptomatic IC hemorrhage, none of the patients developed symptomatic intracerebral hematoma; however, 12 patients (44\%) demonstrated signs of bleeding on the follow-up brain imaging. According to the ECASS II study criteria on hemorrhagic events, ${ }^{14} 9$ of these 12 patients presented with a $\mathrm{PH}$ of grade 1 , and none of them presented with a $\mathrm{PH}$ of grade 2 . The remaining 3 patients had an $\mathrm{HI}$ of grade 1 or 2 . Two patients 

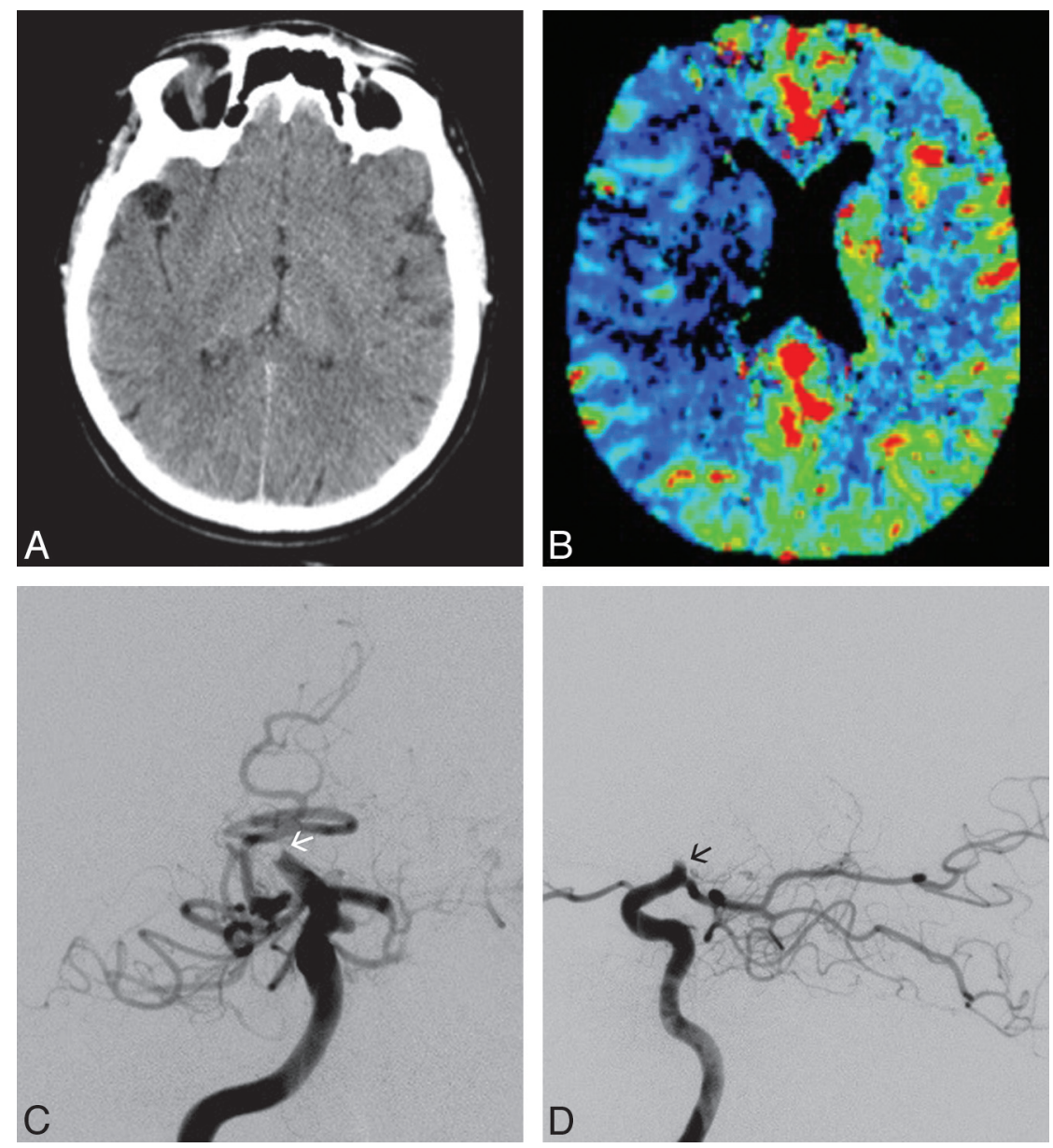

Fig 1. Diagnostic illustration of a patient with acute stroke before treatment with the PS. $A$ and $B$, Axial unenhanced CT scan $(A)$ and CT perfusion cerebral blood flow map (B) obtained 2.5 hours after symptom onset demonstrate only subtle changes in the brain but a significant perfusion deficit in the right MCA territory. $C$ and $D$, Selective right ICA injection angiogram, posteroanterior $(C)$ and lateral $(D)$ views, shows complete thromboembolic occlusion (arrows) of the ICA at the level of the origin of the anterior choroidal artery, which is still filling.

(7\%) became candidates for decompressive craniectomy required to treat mass effect in cases of large infarction.

At 3 months after stroke and interventions, 3 patients had died, resulting in an all-cause mortality rate of $11 \%$. All 3 patients were successfully revascularized to a TICI score of $2 \mathrm{~b}$ or 3. Nevertheless, 2 of them did not show any improvement after intervention and died from the progression of the disease. The third patient improved from an initial NIHSS score of 9 to 3 after thromboaspiration but died from a malignancy before the 3-month follow-up.

Patients showing a good functional outcome at 3 months according to the mRS score $(n=13)$ had either a complete $(n=11[85 \%]$, TICI 3$)$ or a partial $(n=2[15 \%]$, TICI $2 \mathrm{~b})$ revascularization. Inversely, of 14 patients with complete revascularization (TICI 3), 11 (79\%) had a favorable functional outcome at 3 months. There was a significant correlation between the degree of revascularization and clinical outcome $(P=.003)$. According to the statistical analysis, baseline NIHSS scores also affected the clinical outcome: Patients with a good clinical outcome presented with lower NIHSS scores at baseline (median NIHSS, 9 [IQR, 6-14]), compared with patients with a bad outcome (median NIHSS, 17 [IQR, 14-18], $P=.003)$.

Overall, regarding times and delays, symptom onset to procedure time and to recanalization time was longer in patients with favorable outcome, though not significantly (5h, $4 \mathrm{~min}$ versus $3 \mathrm{~h}$, $54 \mathrm{~min}, P=.17$; and $6 \mathrm{~h}, 36 \mathrm{~min}$ versus $5 \mathrm{~h}, 32 \mathrm{~min}$, $P=.07$, respectively), whereas procedure duration was slightly shorter in the group with favorable outcome (1h, 32 $\min$ versus $1 \mathrm{~h}, 42 \mathrm{~min}, P=.47)$. These delays were not significantly influenced by IV or IA rtPA or a combination treatment including IC or cervical carotid stent placement.

\section{Discussion}

Current reported clinical experience reveals that a large thromboembolic occlusion of the IC vasculature is highly resistant not just to IV thrombolysis ${ }^{16}$ but also to other IA pharmacologic and mechanical revascularization modalities. ${ }^{9,17} \mathrm{~A}$ significant clot load affecting the ICA bifurcation (carotid T), M1 segment of the MCA, and the basilar artery is associated with a high morbidity and mortality rate and with a less favorable clinical outcome. ${ }^{18-21}$ It was also demonstrated that early revascularization correlated very well with good clinical outcome. $^{6,7,17}$ The PROACT and PROACT II trials have shown revascularization rates of $58 \%$ and $66 \%$, respectively, with good clinical outcomes of mRS $0-1$ at 3 months in $31 \%$ in the PROACT subjects and of mRS of $0-2$ in $40 \%$ of the PROACT II subjects. Analysis of the MERCI and Multi MERCI trials has shown that the best results were achieved with combined thrombectomy and thrombolytic treatments. With revascu- 

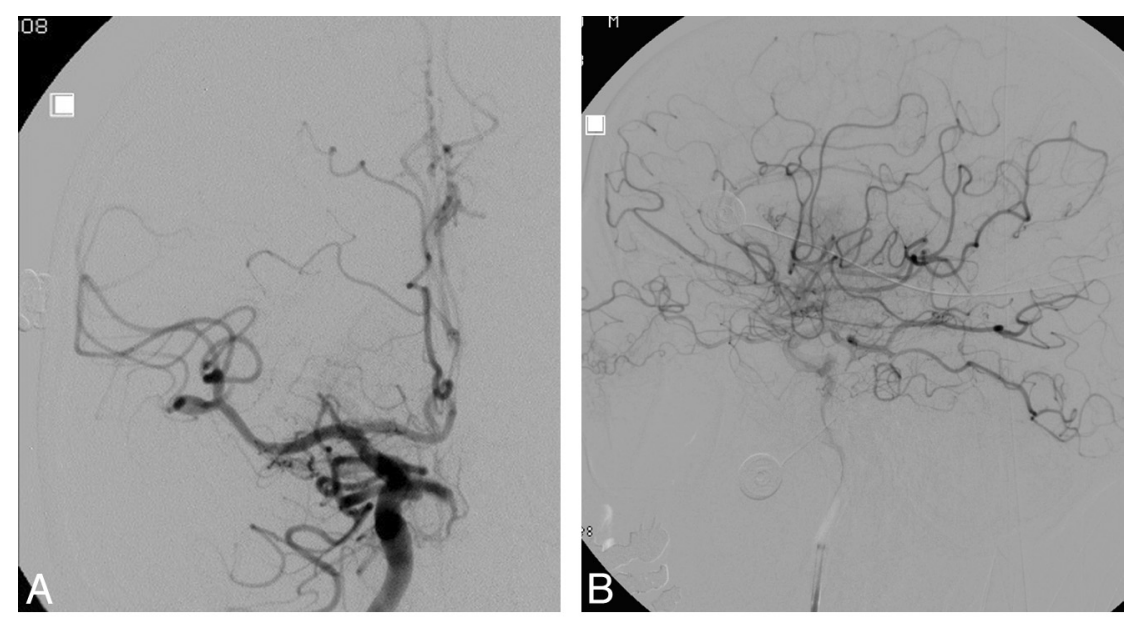

Fig 2. Angiographic illustration of the right ICA after complete revascularization with the PS in the same patient as shown in Fig 1. Posteroanterior $(A)$ and lateral views $(B)$. $C$ and $D$, Axial unenhanced follow-up CT images obtained 24 hours after treatment demonstrate only small putaminal and corona radiata ischemic lesions on the right. The patient had a complete recovery.
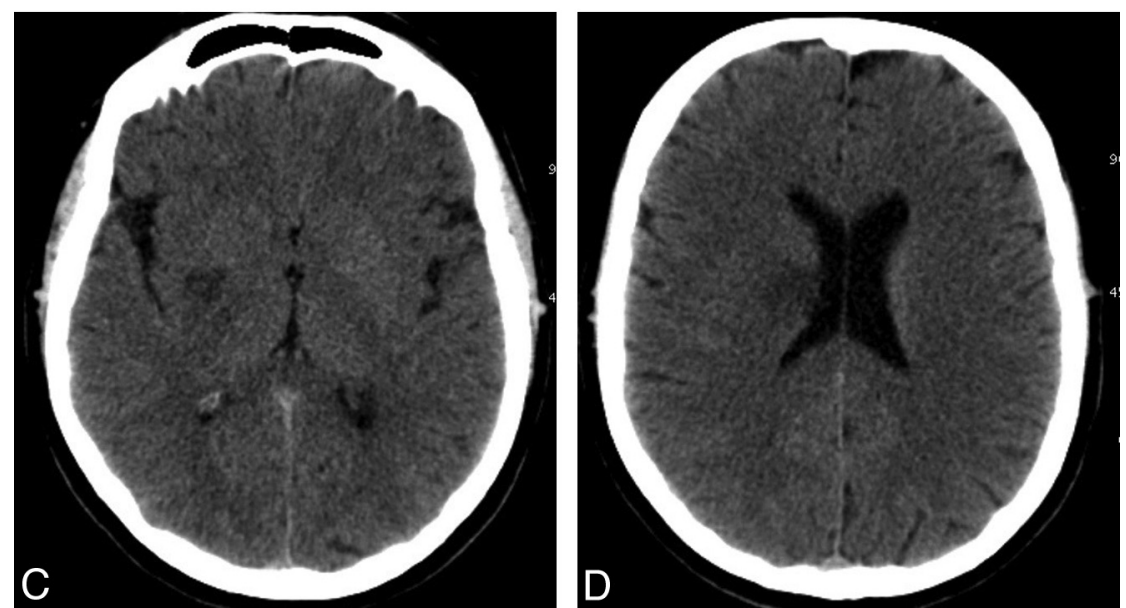

\begin{tabular}{|c|c|c|c|c|c|c|}
\hline $\begin{array}{l}\text { Final } \\
\text { Revascularization }\end{array}$ & $\begin{array}{c}\text { PS } \\
\text { Alone }\end{array}$ & IA rtPA & IC Stent & $\begin{array}{c}\text { Total } \\
(\%)\end{array}$ & $\begin{array}{c}>4 \text {-Point Improvement on NIHSS at } \\
\text { Discharge }(\%)\end{array}$ & $\begin{array}{c}\text { mRS 0-2 at } 3 \text { Months } \\
(\%)\end{array}$ \\
\hline & 1 & 1 & - & $2(7)$ & - & - \\
\hline TICl 1 & - & - & - & $0(0)$ & - & - \\
\hline $\mathrm{TICl} 2 \mathrm{a}$ & 3 & 2 & & $5(19)$ & $2(40)$ & - \\
\hline $\mathrm{TICl} 2 \mathrm{~b}$ & 2 & 3 & 1 & $6(22)$ & $3(50)$ & $2(7)$ \\
\hline $\mathrm{TICl} 3$ & 5 & 7 & 3 & $14(52)$ & $10(71)$ & $11(41)$ \\
\hline Total & & & & $27(100)$ & $15(56)$ & $13(48)$ \\
\hline
\end{tabular}

a Data are the number of patients. - indicates not applicable.

larization rates of $60 \%$ and $70 \%$, a good clinical outcome was achieved in $27 \%$ and $36 \%$ of patients, respectively, in these 2 studies. ${ }^{8,9}$ Both trials provided good evidence that greater revascularization rates resulted in better clinical outcome.

The PS has recently became available as part of the standard of care for acute stroke. This system provides a new promising option for achieving IC vessel revascularization by an aspirationdebulking process. Results from a recent published international multicenter trial evaluating the PS showed a revascularization rate of $100 \%$ (TIMI, 2 or 3 ) in 20 patients with acute stroke in the anterior and posterior circulation. The mean baseline NIHSS score in this series was $21 \pm 8$. Patients were treated within an 8 -hour time window from the onset of symptoms. Of the 20 patients, $7(35 \%)$ had a good functional outcome mRS of $\leq 2$ at 30 days and the mortality rate was $45 \% .^{12}$

In our consecutive case review series, by using the PS alone or in combination with adjunctive IA thrombolysis or IC stent placement, we were able to achieve a revascularization rate of
93\% (TICI, 2 or 3). Complete revascularization was achieved in $52 \%$ of patients. Our analysis revealed that good clinical outcome was best correlated with complete revascularization. After intervention, $56 \%$ of patients experienced a $\geq 4$-point improvement on the NIHSS score at discharge, and $48 \%$ of patients achieved a good clinical outcome at 3 months. The overall mortality rate in this consecutive series was $11 \%$, meaning 3 patients of 27 had died by 3 months. One of the deaths was related to the patient's terminal cancer, which would result in a stroke-related mortality of only $7.4 \%$, far better than expected in such population compared with previously published data. ${ }^{12}$

The relatively good clinical outcome results and the low disease-related mortality rate of our series were probably influenced in part by the comparatively high rate of revascularization achieved but also by the less severe baseline neurologic status of patients as measured by the NIHSS (the score was 14). Patients experiencing a less severe neurologic status despite 
occlusion of a large cerebral vessel may have a good collateral circulation and vascular reserve capacity. ${ }^{22,23}$ We believe this could have contributed significantly to good clinical outcome. However, concerning symptom onset to revascularization time, more surprising in our patient series, there was no significant difference between the subgroups of patients with a good or a bad outcome ( 6 hours 3 minutes versus 5 hours 27 minutes). This finding suggests that collateral circulation and vascular reserve capacity might have a more important role in outcome than the time to revascularization.

In our series, we found no symptomatic intracerebral hemorrhage after treatment according to the ECASS II study criteria. In the PROACT and PROACT II studies, the rates of symptomatic bleeds after endovascular therapy were $15.4 \%$ and $10 \%$, respectively. The MERCI and Multi MERCI trials have shown a sICH rate of $7.8 \%$ and $9.8 \%$, respectively. The Penumbra trial found a sICH rate of $11.2 \% .^{12}$ Apparently, with the advent of endovascular mechanical devices and reduced use of thrombolytics, the rate of sICH has shown a tendency to decline. We believe that having no sICH in our patient series is likely due to the small sample size. Furthermore, one could assume that with limited use of rtPA administered in combination with the thromboaspiration system, the risk of hemorrhage will be reduced. By this technique, locally accumulated potentially deleterious rtPA concentrations could also be aspirated and removed from the vasculature. The low NIHSS score on arrival may also explain the absence of $\mathrm{sICH}^{24} \mathrm{On}$ the other hand, almost half of the patients (12/27) showed some degree of asymptomatic hemorrhagic transformation on control CT.

The average time from the first until the last angiographic study was approximately 100 minutes in this patient series. We do not believe this relatively long duration was due to preparation of the coaxial catheter system, though building up a triple or quadruple coaxial system is more time-consuming than using only a double coaxial system. In our experience, the time needed to prepare such systems is easily regained during the procedure by the efficiency of the system in accessing the target lesions. Furthermore, in our hands, this technique provided a $100 \%$ lesion site access rate compared with the Penumbra trial, in which treatment of 3 patients of 23 had to be abandoned because of accessing difficulties. Most of the working time was spent on the aspiration-debulking manipulations.

Limitations of this study include the relatively small number of patients, the retrospective manner of data collection and analysis, and the concomitant use of IA thrombolysis and IC stent placement as adjuncts to mechanical thrombectomy by the PS.

\section{Conclusions}

The Penumbra thromboaspiration system proved to be very effective for complete revascularization of the cerebral circulation in patients with thromboembolic occlusions of major cerebral vessels. Good clinical outcome was correlated significantly to complete revascularization and the presence of collateral supply. Shorter time from symptom onset to procedure or to recanalization was not associated with better outcome, emphasizing the critical role of neurologic deficit rather than time as a prognostic factor in our population of large-vessel occlusion treated within 6 hours. We believe that the PS has the potential for achieving complete revascularization and improving clinical outcome in acute ischemic stroke with largevessel occlusion.

\section{References}

1. Zaidat OO, Suarez JI, Santillan C, et al. Response to intra-arterial and combined intravenous and intra-arterial thrombolytic therapy in patients with distal internal carotid artery occlusion. Stroke 2002;33:1821-26

2. Tissue Plasminogen Activator for Acute Ischemic Stroke: The National Institute of Neurological Disorders and Stroke rt-PA Stroke Study Group. N Engl J Med 1995;333:1581-87

3. Kim YS, Garami Z, Mikulik R, et al, for the CLOTBUST Collaborators. Early recanalization rates and clinical outcomes in patients with tandem internal carotid artery/middle cerebral artery occlusion and isolated middle cerebral artery occlusion. Stroke 2005;36:869-71. Epub 2005 Mar 3

4. Arnold M, Nedeltchev K, Mattle HP, et al. Intra-arterial thrombolysis in 24 consecutive patients with internal carotid artery T occlusions. J Neurol Neurosurg Psychiatry 2003;74:739-42

5. Rubiera M, Alvarez-Sabin J, Ribo M, et al. Predictors of early arterial reocclusion after tissue plasminogen activator-induced recanalization in acute ischemic stroke. Stroke 2005;36:1452-56

6. Rha J-H, Saver JL. The impact of recanalization on ischemic stroke outcome: a meta-analysis. Stroke 2007;38:967-73

7. Zangerle A, Kiechl S, Spiegel M, et al. Recanalization after thrombolysis in stroke patients: predictors and prognostic implications. Neurology 2007;68:39-44

8. Smith WS, Sung G, Saver J, et al. Mechanical thrombectomy for acute ischemic stroke: final results of the Multi MERCI trial. Stroke 2008;39:1205-12. Epub 2008 Feb 28

9. Smith WS, Sung G, Starkman S, et al. Safety and efficacy of mechanical embolectomy in acute ischemic stroke: results of the MERCI trial. Stroke 2005;36:1432-38

10. Brekenfeld C, Schroth G, Mattle HP, et al. Stent placement in acute cerebral artery occlusion: use of a self-expandable intracranial stent for acute stroke treatment. Stroke 2009;40:847-52

11. Levy EI, Mehta R, Gupta R, et al. Self-expanding stents for recanalization of acute cerebrovascular occlusions. AJNR Am J Neuroradiol 2007;28:816-22

12. Bose A, Henkes H, Alfke K, et al. The Penumbra System: a mechanical device for the treatment of acute stroke due to thromboembolism. AJNR Am J Neuroradiol 2008;29:1409-13. Epub 2008 May 22

13. Higashida RT, Furlan AJ, Roberts $\mathrm{H}$, et al. Trial design and reporting standards for intra-arterial cerebral thrombolysis for acute ischemic stroke. Stroke 2003;34:e109-37

14. Hacke W, Kaste M, Fieschi C, et al. Randomised double-blind placebo-controlled trial of thrombolytic therapy with intravenous alteplase in acute ischaemic stroke (ECASS II): Second European-Australasian Acute Stroke Study Investigators. Lancet 1998;352:1245-51

15. Théron J, Nelson M, Alachkar F, et al. Dynamic digitized cerebral parenchymography. Neuroradiology 1992;34:361-64

16. Saqqur M, Uchino K, Demchuk AM, et al. Site of arterial occlusion identified by transcranial Doppler predicts the response to intravenous thrombolysis for stroke. Stroke 2007;38:948-54

17. Furlan A, Higashida R, Wechsler L, et al. Intra-arterial prourokinase for acute ischemic stroke: The PROACT II study-a randomized controlled trial. Prolyse in Acute Cerebral Thromboembolism. JAMA 1999;282:2003-11

18. Zaidat OO, Suarez JI, Santillan C, et al. Response to intra-arterial and combined intravenous and intra-arterial thrombolytic therapy in patients with distal internal carotid artery occlusion. Stroke 2002;33:1821-27

19. Sims JR, Rordorf G, Smith EE, et al. Arterial occlusion revealed by CT angiography predicts NIH stroke score and acute outcomes after IV tPA treatment. AJNR Am J Neuroradiol 2005;26:246-51

20. Linfante I, Llinas RH, Selim M, et al. Clinical and vascular outcome in internal carotid artery versus middle cerebral artery occlusions after intravenous tissue plasminogen activator. Stroke 2002;33:2066-71

21. Rubiera M, Ribo M, Delgado-Mederos R, et al. Tandem internal carotid artery/ middle cerebral artery occlusion: an independent predictor of poor outcome after systemic thrombolysis. Stroke 2006;37:2301-05

22. Jovin TG, Gupta R, Horowitz MB, et al. Pretreatment ipsilateral regional cortical blood flow influences vessel recanalization in intra-arterial thrombolysis for MCA occlusion. AJNR Am J Neuroradiol 2007;28:164-67

23. Kucinski T, Koch C, Eckert B, et al. Collateral circulation is an independent radiological predictor of outcome after thrombolysis in acute ischaemic stroke. Neuroradiology 2003;45:11-18

24. Singer OC, Berkefeld J, Lorenz MW, et al. Risk of symptomatic intracerebra hemorrhage in patients treated with intra-arterial thrombolysis. Cerebrovascular Diseases 2009;27:368-74. Epub 2009 Feb 16 